\title{
Engineered optical nonlocality in nanostructured metamaterials
}

\author{
Alexey A. Orlov, ${ }^{1}$ Pavel M. Voroshilov, ${ }^{1}$ Pavel A. Belov, ${ }^{1,2}$ and Yuri S. Kivshar ${ }^{1,3}$ \\ ${ }^{1}$ St. Petersburg University of Information Technologies, Mechanics and Optics (ITMO), St. Petersburg 197101, Russia \\ ${ }^{2}$ Queen Mary University of London, Mile End Road, London E1 4NS, United Kingdom \\ ${ }^{3}$ Nonlinear Physics Centre and Center for Ultrahigh-bandwidth Devices for Optical Systems (CUDOS), Research School of Physics and \\ Engineering, Australian National University, Canberra ACT 0200, Australia
}

(Received 1 February 2011; revised manuscript received 11 April 2011; published 12 July 2011)

\begin{abstract}
We analyze dispersion properties of layered metal-dielectric structures, which can be considered as a simple example of nanostructured metamaterials. We demonstrate that, in sharp contrast to the results of the theory of effective media, the layered structure demonstrates strong optical nonlocality due to excitation of surface plasmon polaritons. Such nonlocality can be engineered by changing a ratio between the thicknesses of metal and dielectric layers. Importantly, the nonlocality leads to the existence of an additional extraordinary wave that manifests itself in the splitting of the transverse-magnetic polarized beam refracted at an air-metamaterial interface.
\end{abstract}

DOI: 10.1103/PhysRevB.84.045424

PACS number(s): 78.20.Ci, 42.70.-a, 78.20.Bh

\section{INTRODUCTION}

It is well accepted that the properties of optical composites and nanostructured metamaterials can be described by effective parameters derived under a broad assumption that the structural elements of a composite are much smaller than the wavelength. ${ }^{1}$ The effective medium is an important concept of the homogenization theory based on field averaging, and it provides a physical insight into the optical response of complex microstructured and nanostructured media, being also useful for different types of waves. ${ }^{2}$ However, it was already established that the effective medium models do not provide complete information, and they should be corrected in some cases, e.g., in the recently analyzed case of plasmonic nanorod metamaterials in the epsilon-near-zero regime, where the performance of such structures is affected by nonlocal response. $^{3}$

Here we demonstrate that the dispersion properties of metal-dielectric periodic nanostructured metamaterials are dramatically affected by a nonlocal response due to excitation and coupling of surface plasmon polaritons at the metaldielectric interfaces, so, in many cases, the effective medium approach fails to describe correctly the optical properties of such structures. The difference is dramatic, and it can not be taken into account by small corrections. ${ }^{3,4}$ Nevertheless, we reveal that the strength of optical nonlocality can be engineered in a rather simple way, just changing a ratio between the thicknesses of metal and dielectric layers, so that the medium with equal layers demonstrates weak nonlocality.

The electromagnetic response of periodic layered metaldielectric nanostructures has been a subject of many theoretical and experimental studies. Such structures represent the simplest nanostructured metamaterials, and they were suggested for a number of applications including superlenses with subwavelength resolution, ${ }^{5-7}$ as a simple realization of the so-called hyperlens, ${ }^{8}$ as well as for nanolithography, ${ }^{9}$ optical nanocircuits, ${ }^{10}$ invisibility cloaks, ${ }^{11}$ and even threedimensional negative refraction. ${ }^{12}$ In many cases, the effective medium is a conventional approach for describing optical properties of such structures, and it allows us to introduce the effective permittivities and show that such structures behave as uniaxial metamaterials with the optical axis parallel to the layers.

In this paper, we demonstrate, both analytically and numerically, that the metal-dielectric structures can demonstrate strong optical nonlocality due to the excitation of surface plasmon polaritons, depending on the thicknesses of layers. In particular, we reveal the existence of an additional extraordinary wave, which affects dramatically the scattering of the transverse-magnetic (TM) polarized wave at an airmetamaterial interface, generating two waves with negative and positive refraction, respectively.

The paper is organized as follows. In Sec. II, we discuss the basic results of the effective medium approach that allows us to describe a layered medium as an effective uniaxial anisotropic medium characterized by the permittivity tensor. Section III summarizes the exact results of the transfer-matrix approach, and it provides a detailed comparison with the effective theory for three different cases. In Sec. IV, we discuss some consequences of the effective nonlocal response, in particular, the beam splitting due to an additional branch of the dispersion dependencies. Finally, Sec. V concludes the paper.

\section{EFFECTIVE MEDIUM APPROACH}

We consider a nanostructured metamaterial formed by a periodic layered structure (see the inset in Fig. 1), where metal and dielectric layers have different thicknesses. When the wavelength of radiation is much larger than the thickness of any layer, it is usually assumed that the effective medium approach is valid and the permittivity tensor for a uniaxial anisotropic medium has the following form:

$$
\varepsilon_{\text {eff }}=\left(\begin{array}{ccc}
\varepsilon_{\perp} & 0 & 0 \\
0 & \varepsilon_{\|} & 0 \\
0 & 0 & \varepsilon_{\|}
\end{array}\right), \quad \begin{gathered}
\varepsilon_{\|}=\frac{\varepsilon_{1} d_{1}+\varepsilon_{2} d_{2}}{d_{1}+d_{2}}, \\
\varepsilon_{\perp}=\frac{\varepsilon_{1} \varepsilon_{2}\left(d_{1}+d_{2}\right)}{\varepsilon_{2} d_{1}+\varepsilon_{1} d_{2}},
\end{gathered}
$$

where $\varepsilon_{1}, \varepsilon_{2}$ and $d_{1}, d_{2}$ are the dielectric permittivities and thicknesses of the layers, respectively. 


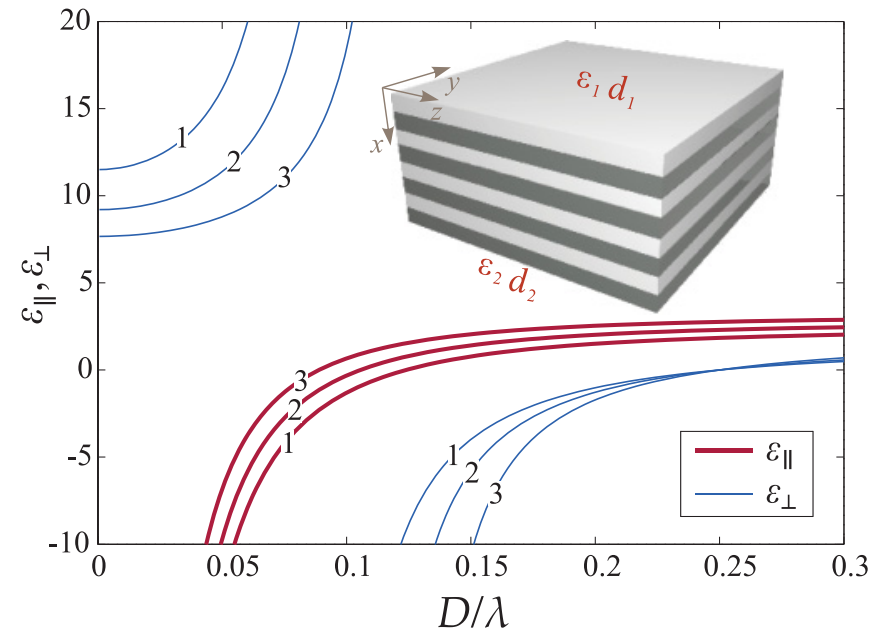

FIG. 1. (Color online) Effective medium model. Permittivity tensor components as functions of normalized frequency for three types of periodic layered metal-dielectric nanostructures with the fixed lattice spacing $D=d_{1}+d_{2}$, but formed by pairs of metal and dielectric layers with the thicknesses (1) $d_{2}=1.5 d_{1}$, (2) $d_{1}=d_{2}$, and (3) $d_{1}=1.5 d_{2}$, respectively.

A dispersion relation for extraordinary waves supported by such an effective uniaxial medium and propagating in the $x y$ plane has the form

$$
\frac{k_{x}^{2}}{\varepsilon_{\|}}+\frac{k_{y}^{2}}{\varepsilon_{\perp}}=\left(\frac{\omega}{c}\right)^{2},
$$

and it establishes a relation between the wave-vector components $k_{x}, k_{y}$ and the frequency $\omega$. Below the plasma frequency, the permittivities of metal and dielectric have different signs, and this ensures a key feature of metaldielectric nanostructures (MDNs): the principal elements of their permittivity tensor can have nearly arbitrary values. For example, if $\varepsilon_{1}=-\varepsilon_{2} d_{1} / d_{2}$, then the structure has very large permittivity $\left(\varepsilon_{\perp} \rightarrow \infty\right)$, and it represents realization of an "epsilon-very-large" (EVL) material. ${ }^{13}$ If $\varepsilon_{1}=-\varepsilon_{2} d_{2} / d_{1}$, then an MDN has near zero permittivity $\left(\varepsilon_{\|}=0\right)$ and is a good candidate for realization of the so-called "epsilon-near-zero" (ENZ) material. ${ }^{14}$ Basically, the EVL and ENZ materials are optical conductors and insulators, which form a basis of metactronics. ${ }^{10}$ If $\varepsilon_{\|}$and $\varepsilon_{\perp}$ have different signs, then the MDN is a typical realization of the so-called indefinite medium. ${ }^{15}$

\section{EXACT DISPERSION RELATIONS}

Now, we apply the standard transfer-matrix approach and analyze, for definiteness, three MDNs formed by layers of metal and dielectric with various thickness ratios, but a fixed total period. The specific structures are illustrated schematically in the insets of Figs. 2(a)-2(c). The dispersion diagrams $D / \lambda\left(k_{y}\right)$ for the three MDNs under consideration shown in Figs. 2(a)-2(c) were computed using two approaches: the effective medium model (approximate approach) and the well-known classical dispersion relation for one-dimensional photonic crystals (exact description).

The properties of such MDNs are investigated at wavelengths that are approximately 10 times larger than the structure period. The permittivity of dielectric is assumed to be constant and equal to $\varepsilon_{1}=4$.6. Permittivity of metal is given by the Drude model: $\varepsilon_{2}=1-\lambda^{2} / \lambda_{p}^{2}$, where $\lambda_{p}=4 D$. The losses in metal are neglected in order to simplify the consideration. The frequency dependencies of $\varepsilon_{\|}$and $\varepsilon_{\perp}$ for three types of MDNs with different ratios of layer thicknesses are shown in Fig. 1. The singularities of $\varepsilon_{\perp}$ and nulls of $\varepsilon_{\|}$ corresponding to EVL and ENZ behavior, respectively, are clearly visible.

The information about dispersion properties of MDN provided by the effective medium model is approximate, and it does not take into account actual periodicity of the structure. The general expressions for $\varepsilon_{\|}$and $\varepsilon_{\perp}$ in Eq. (1) are deduced under quasistatic approximation by using an assumption that the electric field does not vary inside of the layers. If permittivities of the constituent layers are positive, then the requirement that the period of the structure (and thus the thicknesses of the constituent layers) is much smaller than the wavelength of operation ensures negligibly small variation of fields since spatial harmonics propagating inside of the layers have small wave numbers. However, such a reasoning is not valid if materials with negative permittivity (e.g., metals) are involved because the interfaces between materials with positive and negative permittivity support surface plasmon polaritons (SPPs), which form an alternative channel for wave propagation and may cause significant field variation in the layers. The SPPs at the individual interfaces of an MDN exist at the frequencies below the SPP resonance that correspond to condition $\varepsilon_{1}=-\varepsilon_{2}$. It is logical to expect violation of classical homogenization requirement at these frequencies, but only for oblique propagation of waves through the MDN since the
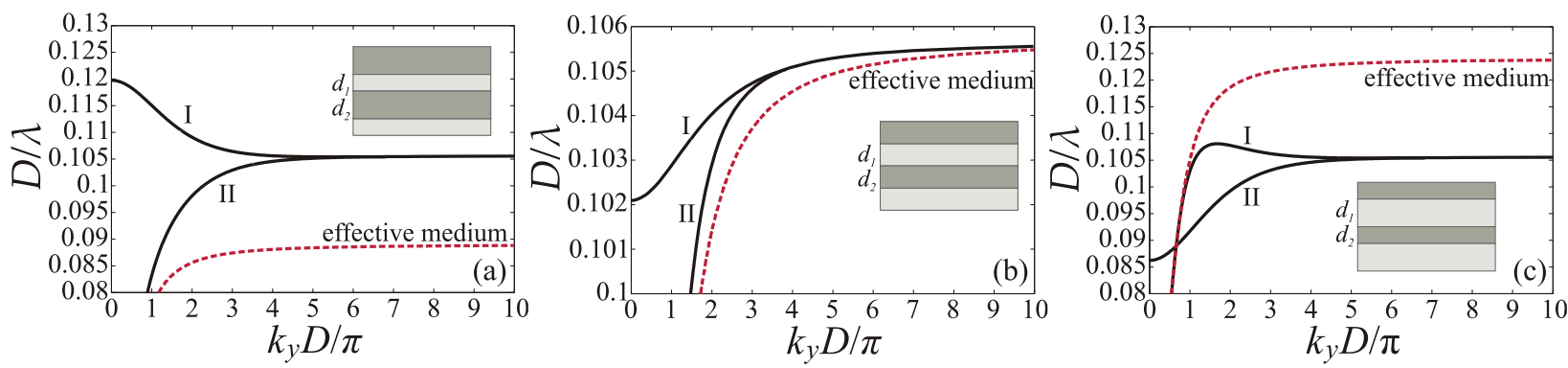

FIG. 2. (Color online) Dispersion diagrams and the geometries of the periodic nanostructures composed of alternating metal and dielectric layers. Thicknesses of the layers are (a) $d_{2}=1.5 d_{1}$, (b) $d_{1}=d_{2}$, and (c) $d_{1}=1.5 d_{2}$. Two dispersion branches corresponding to the actual structures are numbered by Roman numerals. 
waves propagating across the layers can not couple to SPPs. Note that the SPP resonance condition, which corresponds to the strongest confinement of SPPs and thus leads to the strongest variation of fields across the layers, connects only permittivities of the materials that form the interfaces where SPPs propagate, and it does not depend on the thicknesses of the layers.

The dispersion characteristics of actual periodic MDN can be evaluated from Maxwell's equations with imposed periodic boundary conditions. A consideration of the MDN as a one-dimensional photonic crystal leads to the following exact dispersion relation for eigenmodes with transverse-magnetic (TM) polarization:

$$
\begin{aligned}
\cos & \left.k_{x} D\right) \\
= & \cos \left(k_{x}^{(1)} d_{1}\right) \cos \left(k_{x}^{(2)} d_{2}\right) \\
& -\frac{1}{2}\left(\frac{\varepsilon_{2} k_{x}^{(1)}}{\varepsilon_{1} k_{x}^{(2)}}+\frac{\varepsilon_{1} k_{x}^{(2)}}{\varepsilon_{2} k_{x}^{(1)}}\right) \sin \left(k_{x}^{(1)} d_{1}\right) \sin \left(k_{x}^{(2)} d_{2}\right),
\end{aligned}
$$

where $k_{x}^{(1,2)}=\sqrt{\varepsilon_{1,2}(\omega / c)^{2}-k_{y}^{2}}$ are $x$ components of wave vectors in the corresponding layers.

The MDNs with three different ratios of layer thicknesses $(3 / 2,1$, and $2 / 3$, respectively) were chosen in order to demonstrate different behaviors of dispersion curves. In the first case, the SPP resonance (in our case, corresponding to $D / \lambda=0.1055)$ lays over the frequency where EVL behavior is expected $(D / \lambda=0.089)$. In the second case, the frequencies are chosen to be equal. In the third case, the SPP resonance appears well below EVL frequency $(D / \lambda=0.124)$. In all cases, the dispersion curves consist of two branches and have SPP resonance as asymptote if $k_{y} \rightarrow \infty$. However, in the first case, the upper branch corresponds to a backward wave, while the second branch does not. In the second case, both branches correspond to forward waves and coexist at the same frequency range in contrast to the first case. In the third case, the branches cross each other right at the frequency corresponding to ENZ behavior of $\mathrm{MDN}(D / \lambda=0.089)$, and one of the branches has a maximum leading to existence of backward and forward waves simultaneously at the same range of frequencies. The effective medium model in all cases predicts only one propagating wave at all frequencies. The presence of two propagating waves is a consequence of nonlocality, which is caused by SPPs at the interfaces of the layers.

We have verified that the behavior observed for MDNs with the period 10 times smaller than wavelength remains qualitatively the same for MDNs with 2, 4, and 8 times smaller periods (i.e., 20, 40, and 80 times smaller than wavelength). The convergence to an asymptotic line corresponding to SPP resonance becomes weaker (the branches reach this line at larger $k_{y}$ ), but the topology of the branches remains the same. On the other hand, the decrease of period pushes the dispersion curves closer to those predicted by the effective medium approximations for small $k_{y}$. It is interesting that this convergence also depends on the ratio of the layer thicknesses. In the case of equal layers, the convergence is faster than for different layers. However, even for very small period, the correspondence between the results of the effective model and actual dispersion properties of MDNs is not satisfactory,

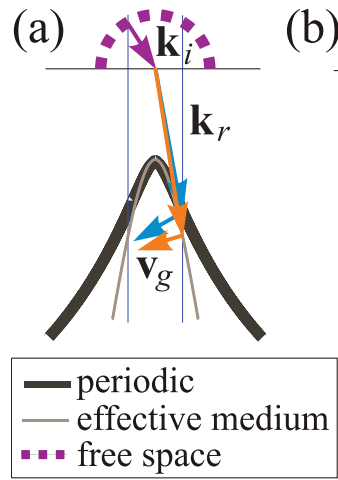

(b)

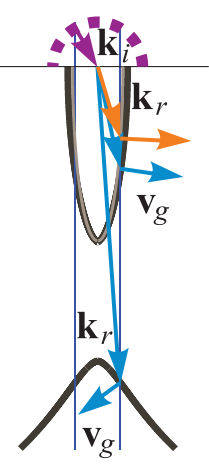

(c)

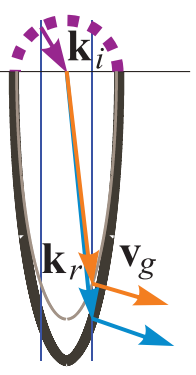

FIG. 3. (Color online) Diagrams of beam refraction at the airMDN interface in the case of Fig. 2(c) at three different frequencies: (a) $D / \lambda=0.085$, (b) $D / \lambda=0.094$, and (c) $D / \lambda=0.106$. Wave vectors $\mathbf{k}_{i}, \mathbf{k}_{r}$ of incident and refracted waves, respectively, and group velocity vectors $\mathbf{v}_{g}$ are plotted by means of the isofrequency contours method. The first Brillouin zone is rendered.

especially for the waves with relatively large $k_{y}$, which are mainly used in the problem of subwavelength imaging.

\section{BEAM REFRACTION AND SPLITTING}

The isofrequency contours can be used for describing the beam refraction at the interfaces separating free space and the layered structure. They allow us to understand the character of refracted waves and find directions of their phase and group velocities. For the three typical cases of isofrequency contours corresponding to the case of $d_{1}=1.5 d_{2}$, the refraction diagrams are plotted in Fig. 3. In the first case [see Fig. 3(a)], there exists only one negatively refracted beam, both for the effective medium model and transfer-matrix method. Here, we have a situation where the effective medium model can be applicable. The same situation is demonstrated in Fig. 3(c), but one positively refracted beam is observed. The simultaneous appearance of ellipse and hyperboliclike contour in Fig. 3(b) leads to beam-splitting phenomena in the metal-dielectric structure. In this case, both negatively and positively refracted TM polarized beams should appear simultaneously.

In order to verify this prediction, we have performed numerical simulations with a commercial full-wave electromagnetic solvers package ${ }^{16}$ for the wave refraction at an interface at different frequencies [see Figs. 4(a)-4(c)]. It should be noted that such simulations are quite hard to accomplish since the beam width is equal to about 10 wavelengths of radiation $\lambda$, with the smallest mesh element reaching $\lambda / 500$. At $D / \lambda=0.085$, only one negatively refracted wave is observed [Fig. 4(a)] and the effective medium model can be applicable. Appearance of an ellipse in the isofrequency contours at $D / \lambda=0.094$ shown in Fig. 3(b) leads to beam splitting [Fig. 4(b)]. The negatively refracted beam still appears but, in addition to it, a positively refracted beam is observed. In this case, the effective medium model is not applicable since it does not predict the presence of the two waves, describing only one of them. At the higher frequency $(D / \lambda=0.106)$, only one positively refracted ray is observed, and this fact is well described by the effective medium model. 

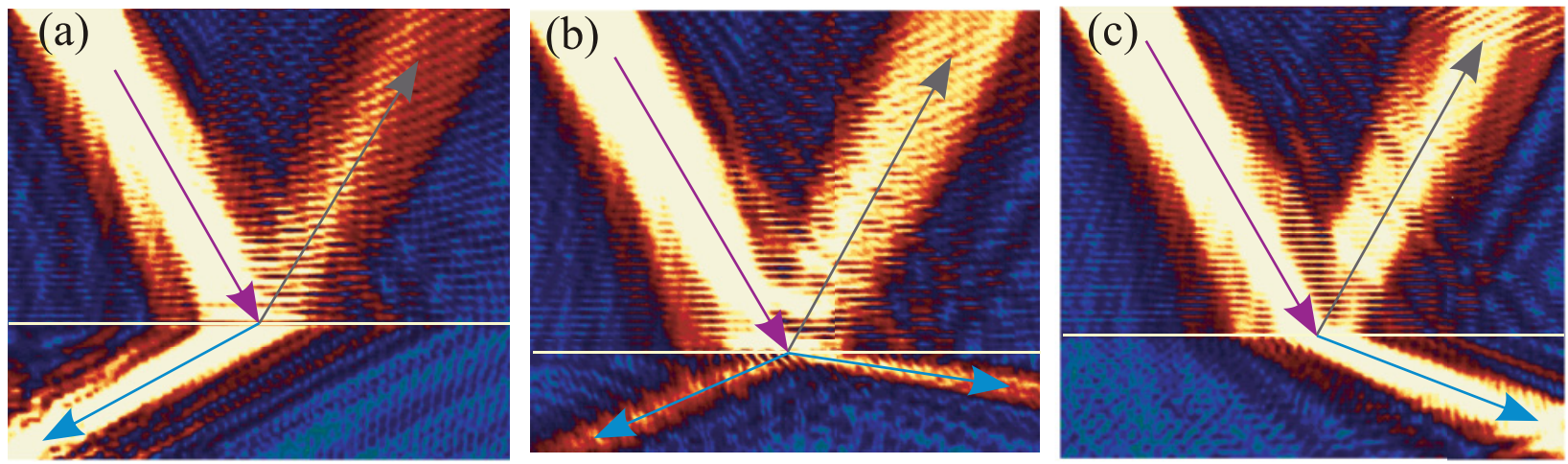

FIG. 4. (Color online) Numerical studies of the beam refraction in the three cases corresponding to Figs. 3(a)-3(c), respectively. Layers are normal to the interface. Incident wave is TM polarized.

Our results call for deeper studies of the applicability limits of the effective medium models, and they may be useful to explain experimental results in more complex settings. In particular, a few years ago, Kozyrev et al. ${ }^{17}$ observed experimentally the waveform splitting into two or multiple beams after the propagation of electromagnetic waves through a slab of a magnetic metamaterial. Such effects can not be described by an effective medium model being characterized by some effective permittivity and permeability. Instead, the observed transmission properties of metamaterials are affected significantly by the internal structure, such that the multiple beam formation observed in the experiments can be attributed to the excitation of magnetoinductive waves.

\section{CONCLUSIONS}

We have studied the dispersion properties of periodic metaldielectric structures by employing the exact transfer-matrix approach and an effective medium model. We have revealed a substantial difference between the results provided by these two approaches, even in the limit when the structure spacing is much smaller than the radiation wavelength. These results question the applicability of the averaged parameters and effective media in the cases when the structure supports surface waves. In particular, we have found two dispersion branches of extraordinary waves instead of one branch predicted by the effective medium model. Importantly, the effective optical nonlocality has been shown to depend dramatically on a ratio between the thicknesses of metal and dielectric layers, and it can be engineered by changing this ratio, with the weakest nonlocality in the case of equal thicknesses.

We have demonstrated that strong nonlocal effects predicted here can be observed as the splitting of the TM polarized wave at an interface between air and metal-dielectric nanostructured metamaterial into two waves with positive and negative refraction. Our results suggest the presence of strong spatial dispersion in many types of nanostructured materials and, in particular, they provide a proof that metal-dielectric nanostructures are inherently nonlocal metamaterials.

\section{ACKNOWLEDGMENTS}

The authors acknowledge support from a mega-grant of the Ministry of Education and Science of Russian Federation (Russia), EPSRC (UK), and Australian Research Council through the Discovery and Center of Excellence Programs (Australia).
${ }^{1}$ D. Smith and J. Pendry, J. Opt. Soc. Am. B 23, 391 (2006).

${ }^{2}$ L. M. Brekhovskikh, Waves in Layered Media (Academic, New York, 1980).

${ }^{3}$ R. J. Pollard, A. Murphy, W. R. Hendren, P. R. Evans, R. Atkinson, G. A. Wurtz, A. V. Zayats, and V. A. Podolskiy, Phys. Rev. Lett. 102, 127405 (2009).

${ }^{4}$ J. Elser and V. A. Podolskiy, Appl. Phys. Lett. 90, 191109 (2007).

${ }^{5}$ S. A. Ramakrishna and J. B. Pendry, Phys. Rev. B 67, 201101 (2003).

${ }^{6}$ P. A. Belov and Y. Hao, Phys. Rev. B 73, 113110 (2006).

${ }^{7}$ X. Li, S. He, and Y. Jin, Phys. Rev. B 75, 045103 (2007).

${ }^{8}$ Z. Liu, H. Lee, Y. Xiong, C. Sun, and X. Zhang, Science 315, 1686 (2007).
${ }^{9}$ Y. Xiong, Z. Liu, and X. Zhang, Appl. Phys. Lett. 93, 111116 (2008).

${ }^{10}$ N. Engheta, Science 317, 1698 (2007).

${ }^{11}$ W. Cai, U. K. Chettiar, A. V. Kildishev, and V. M. Shalaev, Opt. Express 16, 5444 (2008).

${ }^{12}$ E. Verhagen, R. de Waele, L. Kuipers, and A. Polman, Phys. Rev. Lett. 105, 223901 (2010).

${ }^{13}$ J. T. Shen, P. B. Catrysse, and S. Fan, Phys. Rev. Lett. 94, 197401 (2005).

${ }^{14}$ M. Silveirinha and N. Engheta, Phys. Rev. Lett. 97, 157403 (2006).

${ }^{15}$ D. R. Smith and D. Schurig, Phys. Rev. Lett. 90, 077405 (2003).

${ }^{16}$ CST Microwave Studio 2010, CST GmbH [http://www.cst.com].

${ }^{17}$ A. B. Kozyrev, C. Qin, I. V. Shadrivov, Yu.S. Kivshar, I. L. Chuang, and D. W. van der Weide, Opt. Express 15, 11714 (2007). 